\title{
La metacentralidad y sus contradicciones. Tlatelolco como espacio paradoxal de resistencia en la Ciudad de México
}

\author{
Alfonso Valenzuela-Aguilera ${ }^{1}$ \\ Recibido: 22-04-2019 | en su versión final: 17-12-2019
}

Resumen El presente trabajo analiza la importancia de la centralidad en espacios de resistencia social tomando como referente la Plaza de las Tres Culturas en el interior del emblemático Conjunto Urbano Nonoalco-Tlatelolco en la Ciudad de México, lugar en donde tendría lugar la represión violenta de estudiantes en los albores de los XIX Juegos Olímpicos en 1968. A partir de este hecho reflexionamos sobre la importancia de la centralidad en sus dimensiones territorial, temporal, mediática y cultural para la transmisión de mensajes a la población, que en este caso marcó un punto de inflexión importante para la vida social y política del país. Mediante un análisis historiográfico, se examinan los distintos eventos ocurridos en la segunda mitad del siglo XX, en donde se manifiesta la relevancia del caso de estudio en los eventos de 1968. En las protestas se reclamaban las libertades democráticas y Tlatelolco se erige como epicentro urbano desafiando a un urbanismo como instrumento de poder y enfrentándose a una intervención militar que buscaba suprimir contradicciones, demostrar coherencia y reducir un proceso dialéctico a un esquema lógico. Este abordaje revisa la gestación de una ciudadanía emergente en ese periodo, donde una revisión de las relaciones de poder replanteó las políticas de la vida cotidiana urbana. Ante la percepción de las protestas como la creación de un ágora moderno y una nueva apropiación del espacio urbano, concluimos que estos espacios de contestación surgieron a partir de la desesperación y el rechazo de un régimen impositivo y que tuvieron una función instrumental para cimentar la democracia en el tiempo. Se concluye que este evento marca por sí solo el papel que la contestación social tuvo en la creación de una ciudadanía emergente, que a partir de entonces transformó la vida cotidiana de sus habitantes.

Palabras clave: Centralidades; territorio; modernidad; urbanismo

\begin{tabular}{l|l} 
Citación & Valenzuela-Aguilera, A. (2020). La metacentralidad y sus contradicciones. Tlatelolco como \\
espacio paradoxal de resistencia en la Ciudad de México. ACE: Architecture, City and \\
Environment, 14(42), 8224. DOI: http://dx.doi.org/10.5821/ace.14.42.8224
\end{tabular}

1 Doctor, Arquitecto. Profesor Investigador definitivo SNI III, Facultad de Arquitectura, Universidad Autónoma del Estado de Morelos (ORCID: 0000-0003-3238-446X; Scopus ID: 55602640800). Correo de contacto: aval@uaem.mx

ACE, 14 (42) CC BY-ND 3.0 ES | UPC Barcelona, España | La metacentralidad y sus contradicciones. Tlatelolco como espacio paradoxal de resistencia en la Ciudad de México. DOI: http://dx.doi.org/10.5821/ace.14.42.8224 


\title{
Meta-centrality and its Contradictions. Paradoxical Space of Resistance in Tlatelolco, Mexico City
}

Abstract

\begin{abstract}
This paper analyzes the importance of centrality in spaces of social resistance featuring the Plaza de las Tres Culturas located in the emblematic NonoalcoTlatelolco Urban Complex in Mexico City, where violent repression of students took place before the XIX Olympic Games in 1968. Taking this event as a point of departure, the article reflects on the importance of centrality within its territorial, temporal, communicational and cultural dimensions in the transmission of social and political messages to the population. Through a historiographical analysis, the different events that occurred in the second half of the 20th century are examined, and in which the relevance of the case study in the events of 1968 is manifested. During the protests, Tlatelolco served as an urban epicenter where democratic practices were demanded, challenging the urban realm as an instrument of power and challenging military intervention that sought to suppress contradictions, demonstrate coherence and reduce a dialectical process to a logical framework. Through this period, the emergence of a renovated sense of citizenship is redeemed, assessing the configuration of power relations behind urban policies that ruled the daily life of citizens. As protests were perceived as the creation of a modern agora where urban space was reclaimed, we contend that these revolts arose from the despair and rejection of an authoritarian regime and that they were instrumental to cement democracy at the time. It is concluded that this event alone contested the power relations behind democracy, and triggered an emerging citizenship that transformed the daily lives of its citizens.
\end{abstract}

Keywords: Centralities; territory; modernity; town planning

\section{Introducción: la ciudad dentro de la ciudad}

Durante los años sesenta se cuestionaba la expansión metropolitana de la Ciudad de México, traducida en el uso extensivo de suelo urbano que venía aparejado de costosas infraestructuras urbanas, para lo cual algunos urbanistas propusieron aumentar la densidad en zonas de centralidad, con el fin de crear desarrollos habitacionales a gran escala a partir de lo que se denominaba como la "ciudad dentro de la ciudad". Entre dicho tipo de intervenciones se encuentran las unidades habitacionales de Lomas de Plateros, Lindavista-Vallejo, John F. Kennedy, pero destacando por su escala y dimensiones, el Conjunto Urbano Nonoalco-Tlatelolco diseñado por el arquitecto Mario Pani Darqui y que fue concebido para albergar a cien mil habitantes en unidades de interés social inspiradas en el movimiento moderno. Su emplazamiento coincide con la superposición de tres periodos en la historia de México: el prehispánico, el colonial y el moderno, caracterizados todos como etapas de dominación por parte de una serie de élites gobernantes.

La plaza que amalgama estos tres periodos sería testigo de la masacre orquestada contra los estudiantes el 2 de octubre de 1968 y que tendría lugar diez días antes del comienzo de los XIX Juegos Olímpicos. El emplazamiento del proyecto se convertiría en un ejemplo de centralidad espacial por lo que tanto su naturaleza compleja como el contexto espacial resultan ideales para el desarrollo de

ACE, 14 (42) CC BY-ND 3.0 ES | UPC Barcelona, España | La metacentralidad y sus contradicciones. Tlatelolco como espacio paradoxal de resistencia en la Ciudad de México. DOI: http://dx.doi.org/10.5821/ace.14.42.8224 
las presentes exploraciones. El Conjunto Urbano se construyó como referente de los Juegos Olímpicos, haciendo patente la ambigüedad y el significado polisémico de su forma: mientras que para sus creadores quedaba claro que el proyecto se convertiría en un paradigma para resolver el desafío del crecimiento urbano a través de soluciones racionales, para muchos de los residentes la concentración de población perteneciente al sector de menores ingresos en un núcleo urbano de alta densidad urbana no resultó una estrategia sostenible para la ciudad.

Los Juegos Olímpicos modernos fueron reinventados y promovidos por el Barón de Coubertin en 1896, con el objetivo de reunir a deportistas de todo el mundo para celebrar la salud física y la solidaridad humana, en medio de un ambiente internacional que no le fue del todo favorable (Guttmann, 1992, 1994). Este tipo de eventos internacionales servirían como escaparate para el país anfitrión hacia el resto del mundo, pero también la ocasión permitió momentos colectivos de introspección a partir de los cuales se expresó el descontento social, como sucedió en México a principios del siglo XX con las celebraciones oficiales del Centenario de la Independencia, que dieron lugar a la revolución mexicana en momentos en que los contrastes y la inequidad desataron movimientos sociales emergentes, y que habrían de manifestarse en lugares precisos y momentos particulares. Es por ello que en las últimas dos décadas la organización de los Juegos Olímpicos se convirtió en la oportunidad de emprender grandes proyectos de regeneración urbana como un escaparate para ayudar a posicionar a la ciudad en el contexto global (Seúl, 1988; Barcelona, 1992), mostrarse como un Estado moderno (Pekín, 2008), o emprender reformas urbanas importantes (Londres, 2012), e incluso el despliegue internacional ha servido como instrumento para hacer del conocimiento público distintas problemáticas sociales imperantes en el país anfitrión (Ciudad de México, 1968; Atenas, 2004; Río de Janeiro, 2016).

Por otra parte, las olimpiadas han servido como catalizador para la reforma urbana donde las ciudades afirman su carácter, mejoran su estatus y muestran su posición de vanguardia con respecto al resto del mundo (Harvey, 1989). Al convertirse las sedes en el centro de la atención mundial, este tipo de eventos permite a sus residentes mandar mensajes sociales, como fue el caso de Ciudad de México y recientemente de Río de Janeiro. En el presente trabajo se plantea que los acontecimientos represivos que tuvieron lugar en la Plaza de las Tres Culturas en Tlatelolco en 1968, supusieron un punto de inflexión en la relación entre la ciudadanía y el régimen autoritario en México, en donde la violencia dejó entrever las fracturas al interior del gobierno en funciones, por lo que la administración siguiente incluirían voces disidentes en la función pública como una estrategia remedial de inclusión política.

Aun cuando estos hechos develaron los rasgos más oscuros del régimen oficial (represión, prácticas de corrupción, intolerancia política, etc.), también se generaron las semillas de una sociedad civil emergente que, lenta pero constante, comenzaron a transformar el país.

\section{La modernidad urbana y el espacio aséptico}

He aquí lo que quiero decir: no vivimos en un espacio neutro y blanco; no vivimos, no morimos, no amamos dentro del rectángulo de una hoja de papel. Vivimos, morimos, amamos en un espacio cuadriculado, recortado, abigarrado, con zonas claras y zonas de sombra, diferencias de nivel, escalones, huecos, relieves, regiones duras y otras desmenuzables, penetrables, porosas; están las regiones de paso: las calles, los trenes, el metro; están las regiones abiertas de la parada provisoria: los cafés, los cines, las playas, los hoteles; y además están las regiones cerradas del reposo y del recogimiento.

(Foucault, 1966)

ACE, 14 (42) CC BY-ND 3.0 ES | UPC Barcelona, España | La metacentralidad y sus contradicciones. Tlatelolco como espacio paradoxal de resistencia en la Ciudad de México. DOI: http://dx.doi.org/10.5821/ace.14.42.8224 
Tlatelolco es producto de una tradición urbana de la arquitectura moderna en donde el espacio se presenta como un territorio neutro y se intuye en él un potencial para modificar el comportamiento de la gente hasta convertirlos habitantes de la modernidad. Este postulado es justamente a donde se dirige la crítica de Foucault y evidencia la manera abstracta de tratar el espacio urbano por parte de los profesionales del diseño, lo cual coincide con una crítica más aguda sobre la planificación moderna y las implicaciones en la calidad de vida que se originan en las prácticas de zonificación que ésta abandera: "Estas divisiones analíticas no carecen de rigor, pero como ya se ha dicho, el rigor es habitable" (Lefebvre 1991, 35). El desencanto ante la modernidad urbana se traduce en que las divisiones creadas de manera sistemática por las regulaciones de zonificación tienden a clasificar, jerarquizar y homogeneizar artificialmente los espacios. Frente a esto, Lefebvre sugiere que "el estudio de las articulaciones entre los niveles de realidad nos permite demostrar las distorsiones y discrepancias entre niveles, en lugar de difuminarlos" $(1991,105)$.

En el caso de Tlatelolco, encontramos que la proyección de procesos históricos y discontinuidades dentro de la ciudad misma, es decir, que el impulso democrático iniciado en 1968 fue abruptamente interrumpido el 2 de octubre del mismo año en la Plaza de las Tres Culturas, y no obstante, los procesos sociales se inscribieron tanto en el territorio como en el subconsciente de la nación. Tlatelolco sirvió como un recordatorio de las fracturas sociales, las que con el terremoto de 1985 se convertirían en su antípoda, al transformarse en un lugar de solidaridad y en donde se reconfiguró la sociedad civil organizada. En pocos lugares podemos encontrar la dimensión simbólica y nodal del espacio urbano de manera tan evidente como en Tlatelolco, toda vez que la plaza simboliza la esencia misma de la nación mexicana al conjuntar las herencias indígena, española y moderna.

Sin embargo, el mestizaje resulta siempre difícil de definir tanto por su cantidad de matices, como por el hecho de que la cultura local se defina a partir de las rupturas que están en la base de la identidad nacional, definida por la continuidad en el espacio y el tiempo. En ese entendido, el espacio abstracto tiene que ver con los controles, la planificación, la política y la economía, mientras que el espacio social involucra las prácticas sociales, las actividades cotidianas, la apropiación del territorio y el establecimiento de límites siempre cambiantes (Hidalgo et al., 2016, 36). Es por ello que Tlatelolco representa una mezcla entre un sistema urbano impuesto a sus habitantes y el uso colectivo de sus espacios, reminiscente de la idea de la "obra social" creada por los ciudadanos, y es en ese sentido que Lefebvre afirma que los grupos sociales no pueden constituirse o reconocerse como sujetos a menos que generen un espacio, lo cual puede verse de manera simbólica (Lefebvre, 1991).

A lo largo de la historia de las ciudades contemporáneas, la principal herramienta de planificación ha sido la zonificación, la cual surge de la clasificación y división tanto de usos como de funciones en la ciudad. Para domesticar o dominar el espacio, dichos procedimientos de planificación presuponen una visión y determinados valores en el territorio. Es bajo esa racionalidad que se establecen mecanismos de sanción para los infractores del marco normativo o legal, de modo tal que bajo esta perspectiva Lefebvre sugiere que "El poder -que es decir violencia- divide, luego mantiene lo que ha dividido en un estado de separación" (Lefebvre, 1991, 358). Siguiendo este razonamiento, la planificación es una herramienta de conocimiento formal que tiene como objetivo la eliminación de contradicciones, la construcción de una coherencia interna que reduce lo dialéctico a lo lógico frente a la naturaleza compleja de las relaciones sociales, caracterizadas por su diversidad, y en donde "[...] las diferencias perduran o surgen en los márgenes del ámbito homogeneizado, ya sea en forma de resistencias o en forma de externalidades" (Lefebvre, 1991, 373). Frente a lo expuesto, la ciudad se ve obligada a reconocer las diferencias, pero no las relacionadas con el individualismo y la singularidad, sino aquellas que surgen de las luchas reales, conceptuales y vividas. En este sentido, las diferencias no representan una reducción de la realidad ni sirven como un instrumento de poder y dominación, sino que posibilitan el vivir de manera diferenciada, noción que se puso a prueba en nuestro caso de estudio. 


\section{La ciudad moderna: el Conjunto Urbano Nonoalco-Tlatelolco}

Tlatelolco fue concebido en los años sesenta por el arquitecto Mario Pani como la respuesta espacial de la modernidad urbana a las áreas marginadas de la Ciudad de México. La zona del proyecto comprendía una "herradura" de tugurios con una densidad de 500 habitantes por hectárea y la intervención proponía duplicar esta densidad, pero a partir de un esquema vertical. Utilizando la racionalidad Corbusierana, la altura de los multifamiliares permitiría dejar el 75\% del área para espacios verdes mientras que los servicios se alojarían en el nivel inferior de los edificios. Cubriendo un área de cien hectáreas, el Conjunto Urbano Nonoalco-Tlatelolco se proyectó a partir de una serie de edificios habitacionales de distintas alturas colocadas en sentido ortogonal (edificios que variaban entre 4 y 22 pisos), y distribuidas en dos "Supermanzanas" que contrastaban con la escala tradicional y el tejido urbano que tenía esa zona de la ciudad. Al presentar el proyecto, Pani lo planteó como el paradigma moderno y racional, una "ciudad dentro de la ciudad" diseñada para albergar a 100.000 habitantes en 15.000 unidades en donde la sustitución de los tugurios por edificios modernos permitiría "regenerar" la ciudad, resolviendo así las carencias de la capital, tales como el crecimiento urbano desordenado, las áreas residuales deterioradas y las aglomeraciones sin servicios (Pani, 1960).

Mario Pani es considerado como el representante del urbanismo funcional en México. Después de haber cursado sus estudios de arquitectura en la escuela de Beaux Arts de París, en donde adquirió una formación clásica en sentido académico, era imposible sustraerse de la influencia de las ideas de arquitectos como Le Courbusier (con la Ville Radieuse entre otras), o de las propuestas de la ciudad industrial de Tony Garnier. A partir de estos referentes, Pani aplicaría el dogma del movimiento moderno en cada uno de los proyectos que desarrolló a su regreso a México, aprovechando que su familia mantenía vínculos con la élite económica y los círculos de poder político en el país. Fueron precisamente sus lazos familiares los que le permitieron hacerse cargo de proyectos importantes como la conclusión de un par hoteles de lujo en la capital, y más tarde, desarrollar la primera unidad de vivienda en la ciudad bajo el esquema de "supermanzanas" utilizado en Radburn, Nueva Jersey. El modelo de unidades multifamiliares albergaba cuatro veces más personas que lo que la tecnología de construcción tradicional podía ofrecer y por el mismo precio. Es por ello que las propuestas de Pani fueron muy populares entre los políticos mexicanos, tanto por la cobertura extensiva, como por el aura de modernidad implícita en este tipo de desarrollos. Los "Multifamiliares" representaban un cambio en cuanto al esquema de vivienda tradicional, toda vez que los residentes se convertían en propietarios de su vivienda, además de que los proyectos contemplaban áreas comunes para el ocio y el esparcimiento. Sin embargo, en el caso de Tlatelolco, las autoridades excluyeron de manera explícita a los residentes de bajos ingresos, a pesar de que el planteamiento original contemplaba una combinación de grupos socioeconómicos para asegurar un tejido social diversificado. Además, el conjunto urbano debía permitir la integración social mediante los espacios públicos circundantes, fortaleciendo así la centralidad del lugar, pero debido a la falta de mantenimiento de dichos espacios, es que creció el abandono y la desconfianza de los residentes con respecto a su entorno.

Es así que la planificación basada en un crecimiento progresivo de la ciudad pasó a estructurarse mediante grandes proyectos urbanos de carácter monumental, independientes de la escala urbana existente y guardando escasa relación con el entorno local. Sin el menor reparo, Pani comentaba: "cuando se compara el diseño urbano circundante con Tlatelolco, incluso parece una ciudad a una escala diferente [...]" (De Garay, 2000). Es entonces evidente que Tlatelolco no consideró el contexto urbano para que la nueva arquitectura se articulara con el tejido existente, sino que la propuesta se centró en asegurar un 70\% del área del proyecto para espacios abiertos, lo cual generaría por sí misma una sensación de vecindad o algún vínculo identitario sin importar la escala de la operación urbana. Es así que las intervenciones se vincularían en primera instancia con las inversiones de capital a partir de un enfoque inmobiliario, generando con ello una ciudad sobre una base económica

ACE, 14 (42) CC BY-ND 3.0 ES | UPC Barcelona, España | La metacentralidad y sus contradicciones. Tlatelolco como espacio paradoxal de resistencia en la Ciudad de México. DOI: http://dx.doi.org/10.5821/ace.14.42.8224 
inequitativa, en donde los asentamientos irregulares quedarían excluidos de la planificación oficial y los proyectos se concentraron en los sitios en donde generaran el máximo beneficio para el capital.

El Centro Urbano Nonoalco-Tlatelolco se planificó de acuerdo con la tradición "científica" de los estudios urbanos: después de un estudio de diagnóstico que abarcaba dimensiones económicas, sociológicas y geográficas, el proyecto identificaba la necesidad de transformar los asentamientos marginales del centro de la ciudad, buscando recuperar la estructura polinuclear que la ciudad había perdido en la primera mitad del siglo XX. Bajo una visión desarrollista, Pani identificaba a los asentamientos informales como los responsables de la pérdida de convivencia en la ciudad: "[...] la ciudad continúa creciendo a lo largo de las carreteras interestatales, al igual que un enorme pulpo traga los últimos vestigios de la vida social interna" (Pani, 1960, 185). No obstante, durante la construcción de Tlatelolco (1964-1966) se alzaron voces disonantes, entre otras, quienes cuestionaron la idea de transformar los conflictos sociales mediante un esquema de supermanzanas controladas, ordenadas y sobredimensionadas. A este respecto, el entonces alcalde Ernesto P. Uruchurtu conocido como el "Regente de Hierro"- dejó poco espacio para controversias y emprendió distintas transformaciones en la ciudad capital con pocas consideraciones para la historia, las tradiciones o la ciudadanía en general. Pani tampoco sería crítico de sus propios esfuerzos, declarando sin cortapisas que "[Las megaestructuras de gran altura] señalan el camino para las grandes ciudades modernas. Con estos sistemas, la Ciudad de México puede ser cinco veces más pequeña y, al mismo tiempo, permite dedicar alrededor del $80 \%$ de la superficie a parques y jardines, mejorando con ello las condiciones higiénicas de la ciudad" (Pani, 1952, 32).

Vale la pena mencionar el impacto que tuvo Tlatelolco en el entorno urbano de la ciudad, no solo por la escala de la intervención y la morfología arquitectónica, sino también por la ruptura con el pasado. El sitio era conocido por ser un centro prehispánico importante, cercano a la capital azteca de Tenochtitlan. Después de la conquista española, Hernán Cortés asignó el distrito indígena de Tlatelolco al ex-gobernante Cuauhtémoc mientras que la iglesia de Santiago Tlatelolco fue construida en 1527. El proyecto se construyó sobre los vestigios del mercado abierto de Tlatelolco dejando como única reminiscencia los sótanos encontrados en la Plaza de las Tres Culturas. Sin embargo, el uso simbólico de las estructuras prehispánicas y coloniales sirvió a Pani para simular la "fusión" de antiguas tradiciones con las estructuras contemporáneas, representando la continuidad de la cultura mexicana, por así decirlo.

En el número especial de la revista Arquitectura México dedicado a Tlatelolco, aparece sólo una objeción sobre el tema: "[Las operaciones urbanas] deben ir más allá de eliminar las células enfermas mediante cirugía esporádica, pero deben transmitir y responder a la vitalidad del conjunto, convirtiéndose luego en una genuina declaración de justicia social" (Vila, 1966, p. 8).

A los ojos de Pani, el proyecto de Tlatelolco acogería a los habitantes desalojados previamente del cinturón de tugurios, tratándose así de una acción redistributiva mayor, sin considerar entonces el uso instrumental que una obra de tal magnitud representaba para la clase política de la nación. Cabe destacar que desde el gremio de arquitectos y urbanistas existía una preocupación genuina por las cuestiones sociales, de modo que la idea de integración espacial estaban en ese momento en la discusión pública, como destacan los comentarios del arquitecto Vila: "[...] la segregación produce odio, sobre el cual la agitación [social] crece. Mientras [por otro lado], la cordialidad borra o atempera las diferencias sociales inevitables" (Vila, 1966, 101). Por otro lado, publicaciones estadounidenses criticaban los programas de renovación urbana emprendidos por Pani en proyectos similares, argumentando que lo único que se habría logrado con este tipo de proyectos era sustituir los tugurios tradicionales por otros geométricos (Editorial, 1959). A pesar de ello, el arquitecto obvió las críticas y mantuvo magnitud y escala de Tlatelolco, emulando la frase de Daniel Burnham que afirmaba "[...] nunca se logra un gran destino mediante actitudes mezquinas" (Pani, 1966, 8).

ACE, 14 (42) CC BY-ND 3.0 ES | UPC Barcelona, España | La metacentralidad y sus contradicciones. Tlatelolco como espacio paradoxal de resistencia en la Ciudad de México. DOI: http://dx.doi.org/10.5821/ace.14.42.8224 
Tlatelolco es un proyecto paradigmático en varios sentidos: emplazado sobre los dominios de un antiguo reino azteca que más tarde cayó en manos de los conquistadores, y después se convirtió en un tugurio que la renovación urbana intentó despejar. A cuatro años de su inauguración, el geométrico emplazamiento sería el escenario de uno de los principales actos de represión en la historia reciente de México, en donde un incipiente movimiento estudiantil había seguido de cerca el desarrollo de las revueltas francesas en mayo de ese mismo año (donde Lefebvre fue una importante fuente de inspiración), y al igual que los eventos de Tiananmén en China, dicho movimiento presionó por la democracia, el antiautoritarismo y la justicia social en México.

\subsection{La unidad habitacional y las dinámicas de la metrópoli}

El Centro Urbano Nonoalco Tlatelolco fue construido en medio de la acelerada expansión económica y urbana de la capital en la década de los sesenta, al final de la cual la población alcanzó casi 7 millones de habitantes, de los cuales el 70\% tenía menos de 30 años de edad. Aunado a esto, el área urbana se había expandido en más de 100 kilómetros cuadrados en el mismo período, de los cuales el 60\% eran asentamientos informales. En el ámbito urbano, importantes transformaciones tuvieron lugar en esos años, las cuales materializaban contradicciones sociales y puntos de vista enfrentados entre la modernidad y en donde la arquitectura monumental oficial expresaba el poder del Estado. Como una cristalización de los ideales modernos de planificación, Tlatelolco alojó a residentes de bajos ingresos en grandes bloques de construcción a lo largo de espacios verdes rectangulares que incluía la emblemática Plaza de las Tres Culturas. (Figura 1) Este espacio fue diseñado para simbolizar y ensamblar las tres raíces del México moderno: los orígenes mesoamericanos, el pasado colonial representado por la iglesia de Santiago Apóstol y finalmente, el proyecto contemporáneo de vivienda social.

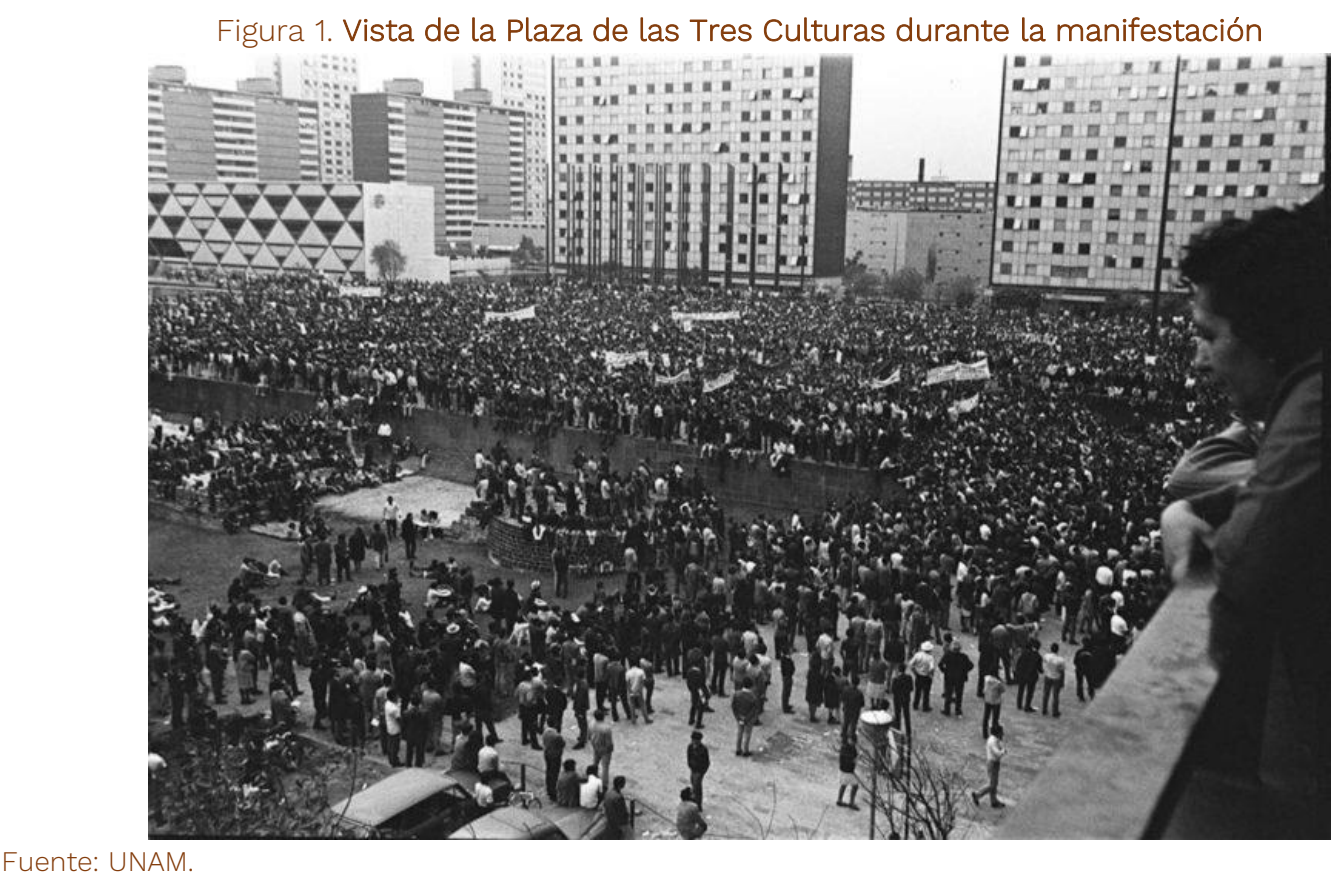

Los Juegos Olímpicos trajeron transformaciones urbanas a la ciudad, pero no los proyectos no respondían a un plan estructurado para articular el tejido urbano, sino que representaron una serie de intervenciones focales y dispersas en el territorio. La planificación urbana no era diferente de la

ACE, 14. (42) CC BY-ND 3.0 ES | UPC Barcelona, España | La metacentralidad y sus contradicciones. Tlatelolco como espacio 
del resto de la estructura política en general, por lo que era jerárquica, autoritaria y directa. Por otra parte, las infraestructuras olímpicas tenían la intención de posicionar a la ciudad dentro de la vanguardia técnica y constructiva de la época que replicaba la fascinación de Kenzo Tange y los Metabolistas por las grandes estructuras. Como se trataba de los primeros Juegos Olímpicos televisados en color, la creación de una imagen de marca fue la clave para la promoción del evento, para lo cual se creó un programa de comunicación y diseño. El reconocido arquitecto y urbanista Pedro Ramírez Vázquez fue nombrado director en jefe de los Juegos, quien se propuso expresar una modernidad cosmopolita en donde, más allá de la campaña integral de imagen y comunicación, se encargó de que las olimpiadas sirvieran para renovar la imagen del Estado, legitimarlo y consolidar así una identidad nacional moderna.

En los Juegos Olímpicos de 1968, la Ciudad de México representaba un quinto de la población total en el país, en un periodo en donde la tasa de crecimiento era del orden del $7 \%$ anual, por lo que dicho evento representaba una excelente oportunidad para mostrar al mundo que la emergente economía mexicana se encaminaba a un circuito económico superior. Para ello, se realizaron importantes inversiones en infraestructura para la capital con el objeto de conectar los diferentes sitios olímpicos, y de manera tangencial, beneficiar a ciertos sectores de la población. La finalización de 16 kilómetros del Circuito Periférico en la parte sur de la ciudad, la construcción de avenidas importantes como el Viaducto Tlalpan $(4,6 \mathrm{Km})$ y la construcción de la primera línea del Sistema de Transporte Colectivo (tren metropolitano) en 1967, establecieron los precedentes para la construcción de modernas infraestructuras de transporte público en los años venideros, y pavimentando así el camino para el modelo desarrollista adoptado por la ciudad contemporánea.

Se calcula que la inversión total fue de alrededor de 80 millones de dólares (Organizing of the Games of the XIX Olimpiad, 1969), y no obstante, la viabilidad económica de la empresa fue cuestionada debido a que los beneficios no resultaron significativos para la población en general, algo que en realidad nunca estuvo entre sus prioridades. Las infraestructuras e instalaciones se terminaron tras dos años de trabajos con el objetivo de atender a más de 6.000 atletas de 130 países, cerca 50.000 visitantes extranjeros, además de 2.000 periodistas y funcionarios de las delegaciones olímpicas. ${ }^{1}$ Según Essex y Chalkley (1998), el Comité Olímpico informó que las instalaciones dispersas crearon una gran tensión en el sistema de transporte público y la gente protestó porque el dinero se invertía en necesidades menos inmediatas, pero más importantes, como la pobreza. Esto es un punto fundamental, ya que señalan en su análisis la profundidad de la crisis social en la que se encontraba el país. Por lo tanto, el problema no sólo fue una cuestión de asignación equilibrada de recursos, sino que existía una democracia emergente que se encontraba en proceso de consolidarse. Como se ha documentado ampliamente, las protestas de los estudiantes fueron mayormente pacíficas, mientras que la represión del gobierno fue extremadamente violenta y enfocada a neutralizar cualquier manifestación de inconformidad.

\subsection{La Plaza de las Tres Culturas como epicentro de la protesta social}

Alrededor de las seis de la tarde del 2 de octubre de 1968 (Figura 2), el ejército mexicano intentó dispersar una concentración de estudiantes que se dio cita en la Plaza de las Tres Culturas en Tlatelolco, después de varios meses de manifestaciones pacíficas. Como parte de la historia oficial, el general José Hernández Toledo, hace el recuento de los hechos en la declaración registrada en el acta del Ministerio Público 54832/68: "Encontrándose de servicio con el batallón a su mando, en la Plaza de las Tres Culturas, alrededor de las 19 horas del día de ayer, (Hernández Toledo) conminó a las personas ahí reunidas a que se retiraran, pero en vez de hacerlo así, dichas personas abrieron

\footnotetext{
${ }^{1}$ Las nuevas instalaciones ocuparon 203 hectáreas, de las cuales 27 se utilizaron para estacionamientos, 143 como espacio abierto y un área construida que suma casi 330.000 metros cuadrados.
}

ACE, 14. (42) CC BY-ND 3.0 ES | UPC Barcelona, España | La metacentralidad y sus contradicciones. Tlatelolco como espacio paradoxal de resistencia en la Ciudad de México. DOI: http://dx.doi.org/10.5821/ace.14.42.8224 
fuego sobre los soldados a su mando, con metralletas tanto desde el piso como desde lo alto de los departamentos del edificio Chihuahua y de otros que se encuentran frente al mismo, al parecer el [edificio] Dos de Abril, por lo que se vio precisado a ordenar al personal a sus órdenes que abriera fuego y, en el momento de ordenar que se cerrara la puerta del edificio Chihuahua, sintió un balazo en la espalda, proveniente del edificio Dos de Abril, a cuyo impacto fue arrojado a una distancia aproximada de dos metros, cayendo boca abajo y arrojando sangre por la boca y que de ahí fue recogido y trasladado a este Hospital Central Militar" (Ortiz Pinchetti, 1988).

Figura 2. Croquis de los disparos de los edificios circundantes a la plaza de las Tres Culturas, 1968

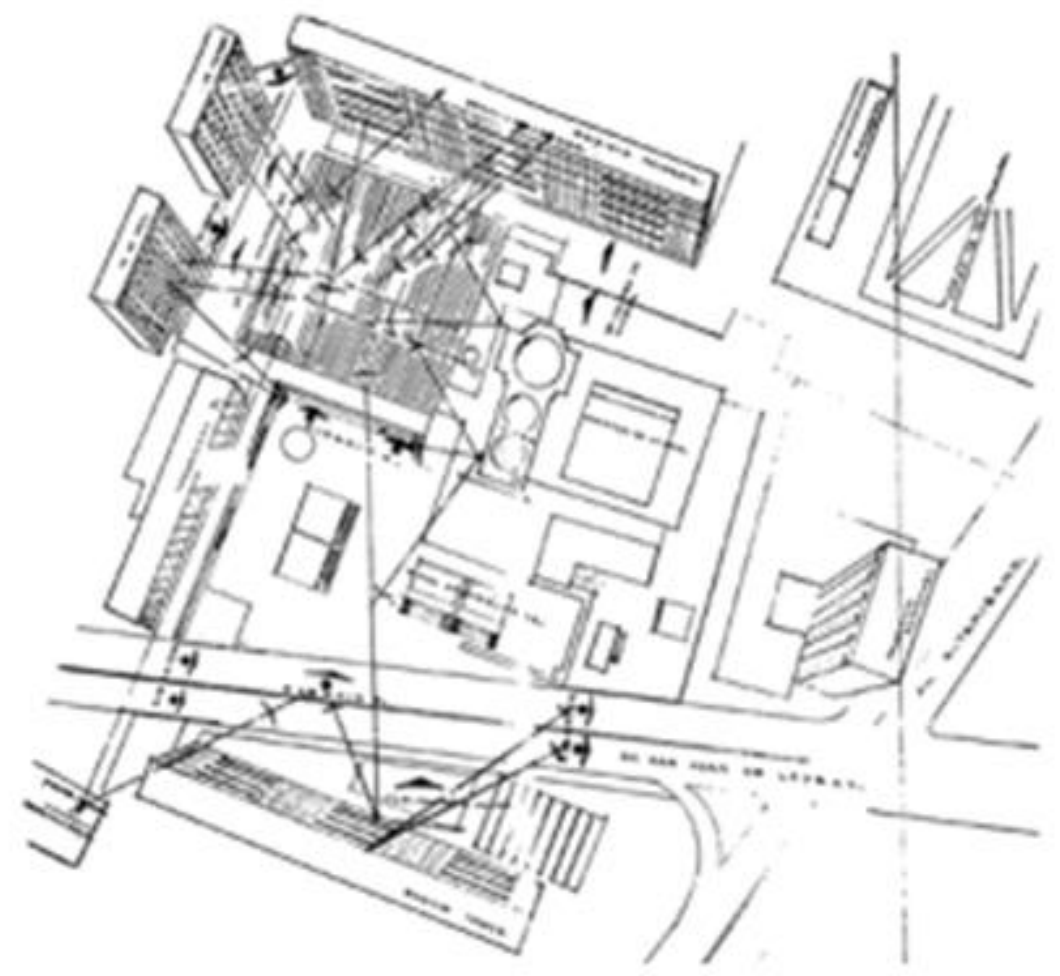

Fuente: Procuraduría General de la República.

No obstante, otras versiones apuntan a que ese día, después de la segunda bengala que fue lanzada desde el techo de uno de los edificios y que coincidió con el patrullaje de un helicóptero militar sobre la zona, los francotiradores que estaban apostados en las azoteas alrededor de la plaza, comenzaron a abrir fuego de manera indiscriminada contra la multitud -pero también contra los soldados- sólo después de que Hernández Toledo recibiera un primer impacto de bala. ${ }^{2}$ En investigaciones posteriores, los francotiradores fueron identificados como miembros del Estado Mayor Presidencial (EMP), un grupo militar de élite bajo las órdenes directas del propio presidente de la república, que tenía la misión de disparar para forzar la respuesta del ejército. Como este último tenía órdenes de comenzar a disparar sólo en el caso que más de cinco de sus soldados causaban baja, los miembros del EMP se aseguraron de que ese fuera el caso. El ejército venía siguiendo las movilizaciones de los estudiantes con anterioridad, de modo que varios departamentos en los edificios que rodean la plaza fueron ocupados semanas antes de la concentración (especialmente los edificios Chihuahua, 2 de

2 Para una narración detallada de los sucesos ver Aguayo, 2015; Scherer y Monsiváis, 1999; Bazán, 2012; Aguayo López Arretche, 1968; Mendoza, 2002.

ACE, 14 (42) CC BY-ND 3.0 ES | UPC Barcelona, España | La metacentralidad y sus contradicciones. Tlatelolco como espacio 
abril, ISSSTE, Molino del Rey, Revolución 1910 e incluso se apostarían el mismo día en el techo de la iglesia colonial). Después de la segunda bengala que marcaría el inicio de los disparos por parte del EMP, la primera reacción de los soldados fue la de proteger a la gente de los francotiradores. (Figura 3)

Figura 3. Planta de los disparos de los edificios circundantes a la plaza de las Tres Culturas, 1968

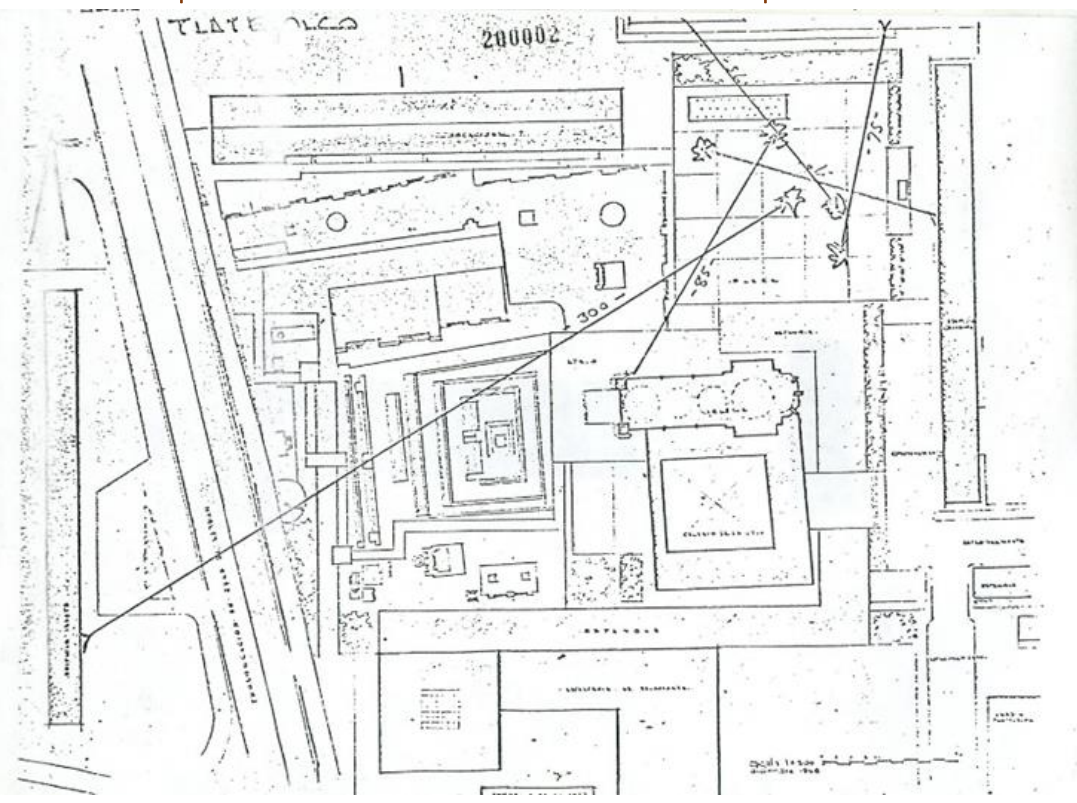

Fuente: Procuraduría General de la República.

Sin embargo, una vez desatado el conflicto, el Batallón Olympia procedió a detener a 400 estudiantes identificados como líderes o miembros de los grupos organizadores para luego ser encarcelados. Llegado ese punto, el ejército ya había bloqueado todas las salidas posibles de los edificios y de la plaza. El tiroteo duró unos 90 minutos, aunque en los primeros diez la población se dispersó al identificar disparos cercanos. Más tarde, oficiales militares de alto rango argumentaron que el ejército había sido utilizado por el gobierno, ya que la batalla no podía atribuirse a un puñado de francotiradores sino a la acción coordinada de varios comandos de élite (Montemayor, 2000, 66). El objetivo de los elementos del EMP fue entonces el generar una escalada de violencia más allá de todo control, utilizando a los estudiantes como el agente provocador, culpando, por tanto, a las víctimas.

Cerca de declarar un Estado de Excepción, el Secretario de la Defensa objetó la suspensión de las garantías civiles sugeridas por el presidente Gustavo Díaz Ordaz, quien probablemente llegó a considerar la instalación del totalitarismo. Un memorándum enviado a los Estados Unidos por el asistente especial para Asuntos de Seguridad Nacional, Walt Rostow, el 5 de octubre de 1968 comenta:

"La CIA cree que las armas empleadas por los estudiantes se pudieron haber obtenido localmente. Y aunque no iniciaron el conflicto, los comunistas mexicanos, trotskistas y castristas capitalizaron los disturbios una vez iniciados y han tomado parte activa" (Montemayor, 2010, 82).

Otro informe de inteligencia evaluó que "[...] como es típico en México, no se pueden encontrar estadísticas sobre el número de víctimas en la batalla del 2 de octubre. Se han recibido informes de hasta 350 muertos. La mejor estimación de la Embajada es que esta cifra está entre 150 y 200"

ACE, 14. (42) CC BY-ND 3.0 ES | UPC Barcelona, España | La metacentralidad y sus contradicciones. Tlatelolco como espacio 10 paradoxal de resistencia en la Ciudad de México. DOI: http://dx.doi.org/10.5821/ace.14.42.8224 
(Montemayor, 2010, 85). Tanto el agente de la CIA asignado a México Winston Scott, como el entonces presidente Díaz Ordaz eran anticomunistas declarados, de modo que las movilizaciones de los estudiantes les parecían una clara evidencia de que podía estar en marcha una intervención de Cuba y China para desestabilizar el país. Aun si no existían evidencias para sugerir que la CIA sabía que el operativo iba a tener lugar en Tlatelolco, ciertamente la agencia contribuyó a crear un clima de represión hacia el movimiento. Se comentaba en un cable desclasificado que: "Agentes del FBI estadounidense en la Ciudad de México que trabajaron en estrecha colaboración con [Winston] Scott informaron que los estudiantes trotskistas habían formado un grupo armado llamado "Brigada Olimpia” para provocar un ataque. Estos estudiantes supuestamente estaban conectados con comunistas guatemaltecos y habrían disparado los primeros disparos. [...] Una fuente dijo que planeaban explotar transformadores para interferir con los eventos olímpicos y confiscar autobuses que transportaran atletas olímpicos." (National Security Archive, 99-20, 68).

\section{La amenaza roja}

"Estos grupos de un modo intuitivo encuentran que nuestro desarrollo político y social no corresponde al progreso económico. [...] No se trata de una revolución social, aunque muchos de sus dirigentes sean revolucionarios radicales, sino de realizar una reforma en nuestro sistema político. Si no se comienza ahora, la próxima década será violenta."

(Octavio Paz, 1968, p. 11)

La premonición de Octavio Paz al diario Le Monde en 1968 coincidía con los temores de la Agencia Central de Inteligencia ( $\mathrm{CIA}$ ) en el sentido de que los Estados Unidos no quería correr ningún riesgo de que el comunismo se estableciera en su patio trasero, por lo que la agencia se infiltró en el gobierno hasta los más altos niveles. Según información desclasificada de la agencia (Informe operacional de LITEMPO del 1 al 31 de octubre de 1963), no sólo los altos funcionarios estaban actuando como informantes sino también el propio presidente Gustavo Díaz Ordaz, así como su sucesor, el secretario de Gobernación Luis Echeverría Álvarez. Después de negociaciones políticas que datan de una década previa, el presidente Vicente Fox acordó en 2002 abrir cientos de miles de documentos clasificados relacionados con la masacre de Tlatelolco y firmar una ley federal de libertad de información. Estos archivos incluían documentos desclasificados de los archivos secretos de la CIA, el Pentágono, el Departamento de Estado, el FBI y la Casa Blanca. Según Kate Doyle, encargada del Archivo de la Seguridad Nacional de Estados Unidos.

"Fox también ordenó la apertura de una extraordinaria colección de registros gubernamentales producidos por los servicios de inteligencia y militares de México durante décadas de violencia patrocinada por el Estado, desde la década de 1960 a la década de 1980" (National Security Archive, 204).

Posteriormente, el presidente Fox nombró a un fiscal especial para presentar cargos penales contra los que resultaran responsables, pero como se podía anticipar, el caso no fue más allá de lo que los documentos desclasificados ya habían mostrado. Aparentemente, la embajada de los EE. UU. dudaba que los estudiantes fueran capaces de representar una amenaza real para el gobierno y, de alguna manera, desconocían que la verdadera política iba más allá de sus informantes clave. Un comunicado del embajador Fulton Freeman al Departamento de Estado explicaba: "El gobierno tiene diversos medios para evaluar e influir en la opinión de los estudiantes, y se ha mostrado capaz y dispuesto, cuando el malestar supera lo que considera límites aceptables, a tomar medidas decisivas, hasta la fecha, con efectos saludables. [...] además, los trastornos de los estudiantes, a pesar de la amplia publicidad que reciben, simplemente carecen de fuerza para crear una crisis nacional" (National Security Archive, 99,1).

ACE, 14 (42) CC BY-ND 3.0 ES | UPC Barcelona, España | La metacentralidad y sus contradicciones. Tlatelolco como espacio paradoxal de resistencia en la Ciudad de México. DOI: http://dx.doi.org/10.5821/ace.14.42.8224 
Dado que el gobierno mexicano estaba previendo disturbios sociales durante los Juegos Olímpicos venideros, las fuerzas del ejército mexicano pidieron al Pentágono material de entrenamiento antidisturbios, armas, municiones y radios militares. La embajada, sin embargo, mantuvo la idea de que el Partido Comunista y la Embajada Soviética estaban tramando tácticas militantes como un cable secreto muestra que apenas dos meses y medio antes de los acontecimientos. Comenta Kate Doyle, quien es también directora del Archivo del Proyecto México: "Cuando estalló la crisis de Tlatelolco, la estación de la CIA en México no pudo cumplir con su cometido [...] como resultado, la agencia deslindó al partido gobernante de México de la responsabilidad de la masacre, entregando a Washington un informe confuso y engañoso de los eventos" (National Security Archive, 204).

Según documentos de la CIA, Luis Echeverría Álvarez había formado un grupo de operaciones estratégicas para abordar el conflicto de los estudiantes, pero no estaban seguros de cómo abordarían las protestas y las nuevas intervenciones. Estos registros dejan claro que los informantes estaban infiltrados a lo largo de toda la estructura del gobierno: el Secretario de Defensa Nacional, la Oficina del Presidente, altos funcionarios, la administración universitaria y entre los mismos estudiantes, en colaboración con la oficina de la CIA en la Ciudad de México. La conmoción social se estaba gestando y la estación de la CIA tuvo que reconocerlo ya por el 9 de septiembre de 1968, afirmando que: "Esta experiencia ha demostrado que el gobierno y el Partido Revolucionario Institucional (PRI) no posee el poder y el control casi total sobre el comportamiento público que existía anteriormente. Si bien hay pocas dudas de que los estudiantes mexicanos han sido influenciados por los levantamientos estudiantiles en los Estados Unidos y Europa, los recientes disturbios estudiantiles han sido una nueva experiencia para México y pueden proporcionar una indicación de lo que vendrá. El viejo orden está pasando, y [...] los estudiantes han descubierto que pueden ser un elemento significativo en el proceso de toma de decisiones de la nación, y ya no están contentos con la actitud condescendiente del gobierno"(National Security Archive, 204).

Dado que la Agencia Central de Inteligencia de los Estados Unidos consideraba que los estudiantes estaban más allá de la actitud paternalista del gobierno y que se habían convertido en una parte importante del proceso de toma de decisiones, la agencia vería esta conciencia democrática emergente como una amenaza real, ya que consideraban que el gobierno mexicano carecía del poder suficiente para mantener a la población bajo control. Sin embargo, inmediatamente después de los eventos de Tlatelolco, un alto funcionario de dicha agencia manifestaba: "Creemos que es importante evitar cualquier indicación de que no tenemos confianza en la capacidad [del Gobierno de México] para controlar la situación" (National Security Archive, 204). Los intereses geopolíticos del vecino del norte estarían siempre en la base de su estrategia continental y más allá de la defensa de valores democráticos, el control de los ciudadanos resultó siempre clave para el funcionamiento de las relaciones económicas y políticas con México. No obstante, una semana más tarde el Departamento de Estado Norteamericano (DEN) reflexionó sobre los acontecimientos argumentando que: "Parece poco probable que el PRI pueda brindar una solución fundamental al problema sin cambiar la extendida convicción de que está atrincherado, estancado y sirviendo a sus propios intereses. A los estudiantes debe convencérseles de que, a pesar de la enorme corrupción y deshonestidad que se han convertido en el sello distintivo del PRI, el partido sigue siendo, o volverá a ser, una fuerza vital para el cambio político y social, así como para el crecimiento económico. El liderazgo actual no parece estar dispuesto a comprender la magnitud del problema de la alienación estudiantil, así como a aceptarlo como una seria advertencia de que el partido no responde a las necesidades legítimas de un segmento cada vez más visible de la sociedad mexicana" (National Security Archive, 204).

Aun pareciendo un cambio arriesgado, el DEN reconocía la importancia del mensaje que los estudiantes estaban transmitiendo a la sociedad, sino también que las necesidades de la población estaban siendo descuidadas, especialmente las relacionadas con la libertad de expresión y las prácticas democráticas en el país. En un cable de la Embajada Norteamericana fechado el 27 de julio

ACE, 14 (42) CC BY-ND 3.0 ES | UPC Barcelona, España | La metacentralidad y sus contradicciones. Tlatelolco como espacio 12 paradoxal de resistencia en la Ciudad de México. DOI: http://dx.doi.org/10.5821/ace.14.42.8224 
de 1968, una evaluación de los enfrentamientos entre fuerzas públicas y estudiantes del día anterior, revelaba la manera en que la policía utilizaba los disturbios como una excusa para irrumpir en las oficinas del Partido Comunista (PCM) para arrestar a varios líderes y apoderarse de sus archivos. No sólo se perseguía la disidencia social, sino incluso la oposición política, la cual tenía cada vez menor cabida en la estructura monolítica del partido oficial en México (National Security Archive 59, 23-28). Si bien la embajada reconocía que la desaceleración de la expansión industrial, el creciente desempleo y la expansión de la pobreza podían generar disturbios en un futuro, calculaba como poco probable que la situación estudiantil alcanzara proporciones críticas al menos en los próximos años" (National Security Archive 59, 13-28).

Unos meses antes, la embajada observó con alarma la evolución de la agitación social después de los enfrentamientos entre la policía y los estudiantes de julio y agosto de 1968, en donde murieron cuatro estudiantes: "Como en el pasado, el grado de culpabilidad comunista es ambiguo. Los disturbios de julio a agosto han implicado hasta ahora niveles más altos de violencia por parte de los estudiantes, un número sin precedentes de personas involucradas y un mayor grado de animadversión contra el gobierno central sin precedentes". Incluso a fines de agosto, la embajada informó que el gobierno de Díaz Ordaz había adoptado una estrategia conciliadora para no intervenir en las protestas, pero detectaba sin embargo la existencia de "agentes del gobierno detrás de las escenas que intentan dividir y debilitar el apoyo de los líderes de la huelga extremista" (Document 10, 1968).

De este modo, quedaba como trasfondo la "guerra sucia" emprendida por el gobierno de Díaz Ordaz, pasando a la ofensiva para descarrilar al movimiento estudiantil. Con la apertura de frentes físicos y psicológicos, el gobierno conminó a la prensa a tomar medidas editoriales más duras contra los estudiantes y emplear el aparato de seguridad con fines represivos. Como sugería otro cable: "El Gobierno de México acepta implícitamente la consecuencia de que esto ocasionará víctimas. Los líderes de la agitación estudiantil han sido y están siendo detenidos [...]". Lo anterior parecería indicar que el gobierno estaría manejando un doble discurso y sin embargo, existen versiones acerca de que, en realidad, existían pugnas políticas entre los miembros del gabinete de modo que mientras empleó por un lado un discurso conciliador, por el otro se ejercieron acciones de represión violenta y velada.

En ese momento, una de las notas de información reservada de la Oficina de Inteligencia e Investigación (INR) del departamento de Estado norteamericano informó del malestar del presidente Díaz Ordaz después de haber sido insultado públicamente durante la manifestación masiva en el Zócalo el 27 de agosto. Derivado de ello, el INR cuestionaba la decisión del régimen de volver a la represión en lugar de negociar, ya que las demandas de los estudiantes no parecían ser excesivas. Sin embargo, los gobiernos del partido oficial eran esencialmente autoritarios y no estaban dispuestos a ceder ante ninguna demanda, posiblemente porque estaba completamente fuera de lugar que un gobierno permitiera que cualquier sector de la sociedad mexicana desafiara su autoridad (Document 32, 1968).

La práctica del autoritarismo puso de manifiesto en la ocasión del informe presidencial sobre el Estado de la Nación, en donde Díaz Ordaz declaró que no permitiría que la figura presidencial fuera desacreditada y prometió que la agitación continuada sería reprimida. Afirmando, además, que la pérdida de respeto por el presidente "crearía graves peligros dentro del sistema político mexicano". El principal peligro para el partido gobernante dominante era, por supuesto, la democratización del país, la creación de mecanismos para lograr la legitimidad y la apertura de otras opciones además del Partido Revolucionario Institucional. La sociedad comenzaba a responder al llamado para transformar el entorno político del país, lo cual se hizo patente en el discurso que el rector del Colegio de México pronunció después de que sus instalaciones fueran ametralladas. En él, realizó severas

ACE, 14 (42) CC BY-ND 3.0 ES | UPC Barcelona, España | La metacentralidad y sus contradicciones. Tlatelolco como espacio paradoxal de resistencia en la Ciudad de México. DOI: http://dx.doi.org/10.5821/ace.14.42.8224 
críticas contra el presidente y atribuyó el malestar de los estudiantes a las condiciones de pobreza en todo el país (Document 14, 1968).

De acuerdo con una conversación entre el profesor universitario Víctor Torres Arriaga con Robert Service -funcionario de la Embajada Norteamericana- comentaba que: "las tácticas represivas utilizadas por el gobierno para contener a los estudiantes se basaban en el miedo a permitir que los estudiantes tuvieran una existencia política verdaderamente independiente". Ningún periódico estudiantil, por ejemplo, "tenía permitido publicar abiertamente y sin controles". Torres argumentaba: "nunca he visto a los estudiantes tan determinados o unificados", y a pesar de los eventos del 2 de octubre, pronosticó que la mayoría de los estudiantes se unirían finalmente al sistema al cabo de unos dos años de haber terminado la carrera. Sin embargo, en una nota de inteligencia previa, los analistas del INR preveían tanto la inevitabilidad de la violencia en los siguientes días, como la posibilidad de que estos eventos generaran más disidencia en el futuro: "El PRI, el partido oficial que ha gobernado México durante casi 40 años, no está acostumbrado a que ningún sector de la sociedad desafíe su autoridad. Los estudiantes, sin embargo, han demostrado que el gobierno y el partido, aunque poderoso, no son invencibles. La lección no se perderá por completo con aquellos grupos que no estén completamente satisfechos con el statu quo" (Document 35, 1968).

Al día siguiente de la masacre de Tlatelolco, el Departamento de Estado atribuyó los eventos a una reacción excesiva por parte de las fuerzas de seguridad ante las provocaciones de estudiantes extremistas. Sin embargo, fueron vistos como un golpe importante para el régimen de Díaz Ordaz y una amenaza real para el éxito de los Juegos Olímpicos, por lo que la CIA informó que el incidente de Tlatelolco planteaba interrogantes sobre la capacidad de México para brindar la seguridad necesaria durante las olimpiadas. Este cable cita a "observadores entrenados" que creían que los estudiantes instigaron el incidente y señala que el gobierno mexicano estaba decidido a evitar una interrupción de los Juegos Olímpicos tanto en la ciudad como en el exterior. "Todos los comandantes de la zona militar ahora tienen autoridad para moverse contra los estudiantes desordenados en las provincias sin consultar con la capital" (Document 77, 1968). De acuerdo con un análisis de los disturbios estudiantiles en América Latina, los trastornos en la capital mexicana habían sido los peores en la región en dicho periodo. La continua violencia demostraba una insatisfacción profunda y generalizada con el gobierno de ese país, dañando severamente la reputación de México como el "país más estable y progresista de América Latina" (Document 43, 1968).

Es interesante comparar esta evaluación con un informe especial de la CIA sobre las condiciones de seguridad en el vecino país, escrito varios meses antes de la primera ola de protestas estudiantiles y donde describen al país como un modelo de estabilidad, con el presidente Díaz Ordaz firmemente en el control y con un partido gobernante que "prácticamente monopoliza la política mexicana" (Document 44, 1968). Un mes después de los eventos de Tlatelolco, la embajada norteamericana visualizaba tres posibles escenarios con respecto a los estudiantes: el primero, en donde las diferencias se resuelven amistosamente entre los estudiantes y el gobierno; el segundo, donde continúa el estancamiento tenso; y el tercero, donde aumenta la violencia considerablemente. Sin embargo, como ha sido costumbre, la embajada no dudaba en recomendar que, en caso de un aumento de la violencia, "Estados Unidos debería estar preparado para mostrar su respaldo al gobierno de Díaz Ordaz, ofreciendo un mayor apoyo financiero y económico" (Document 25, 1968).

Si bien la atmósfera general aún era tensa cuando comenzaron los Juegos Olímpicos, el gobierno mexicano fingió ante los ojos del mundo que todo había vuelto a la normalidad, distribuyendo miles de entradas gratuitas a la ceremonia inaugural a personas conocidas por ser leales al régimen para asegurar que el discurso del presidente fuera aplaudido, y para reducir el número de boletos disponibles para los estudiantes. La CIA también informó que muchos académicos e intelectuales temían que comenzarían a ser perseguidos después de las Olimpiadas y que habían iniciado a buscar

ACE, 14. (42) CC BY-ND 3.0 ES | UPC Barcelona, España | La metacentralidad y sus contradicciones. Tlatelolco como espacio paradoxal de resistencia en la Ciudad de México. DOI: http://dx.doi.org/10.5821/ace.14.42.8224 
citas en el extranjero (Document 79, 1968). Además, los funcionarios mexicanos se preparaban para la violencia futura. "Dos unidades de ejército de 1.500 hombres están en formación para el uso en caso de más violencia, y es probable que el gobierno adopte una dura política de represión si fracasan sus moderadas tácticas conciliatorias" (Document 80, 1968). Los eventos acaecidos en el Conjunto Urbano Nonoalco-Tlatelolco adquirieron entonces una resonancia mayor con las olimpiadas, un evento en el cual la importancia de la Ciudad de México se magnificaba a escala mundial y desde donde cualquier mensaje vendría cargado de simbolismos sobre la madurez democrática del país. El día después de los hechos, sólo la Revista ¿Por qué?, dirigida por el periodista Mario Menéndez Rodríguez, publicó las fotos de la matanza, por lo que fue encarcelado en la prisión de Lecumberri, sus oficinas demolidas y después él mismo sería desterrado del país. El resto de la población permaneció conmocionada y sobrecogida e incluso temiendo el regreso de un régimen militar totalitario, a tal punto que más tarde tuvo que ser negado por el ejército. Diez días después, la llama olímpica se encendió en una ceremonia magnífica, mientras los medios ocultaban la reciente masacre.

Finalmente, Tlatelolco habría de vivir otro punto de inflexión el 19 de diciembre de 1985, cuando un terremoto de 8.1 grados Richter devastó sectores completos del centro de la Ciudad de México y donde varios edificios del Conjunto Urbano colapsaron o sufrieron daños estructurales. El gran conjunto urbano de Mario Pani revelaría los efectos de la falta de mantenimiento y de atención a señales que mostraban ya los edificios y que no fueron atendidas, como el hecho que algunas columnas soportaban tres o cuatro veces más carga que la que podían absorber. Similar a la impresión de la demolición del conjunto de vivienda social de Pruitt-lgoe en St. Louis Missouri en 1972, los edificios revelaron las malas prácticas y la corrupción imperante a lo largo de los años. De todo el conjunto, un edificio colapsó en su totalidad más tarde tuvieron que demolerse ocho edificios más y recortar otros cuatro en altura. Este centro urbano, con una población mayor que la de muchas capitales europeas, pasaría por una dura prueba con el terremoto, particularmente porque la intervención del gobierno fue descoordinada y tardía, ante lo cual la ciudadanía respondió de manera admirable demostrando vínculos de solidaridad nunca antes vistos. En su crónica de los eventos, el escritor Carlos Monsiváis sugiere que en ese momento la sociedad construyó su concepto de sociedad civil: "Por eso, desde el 19 de septiembre, [la] sociedad civil significa para diversos sectores, [el] esfuerzo comunitario de autogestión y autoconstrucción, [así como un] espacio al margen del gobierno y de la oligarquía empresarial" (Monsiváis, 1986, 11-24).

La sociedad mexicana, en su largo camino hacia la democracia, la participación y la madurez política ha afrontado grandes retos. Este desastre natural aceleró la formación de un sentido de civilidad surgido cuando el gobierno mostró sus limitaciones para responder a la emergencia. Las personas mismas descubrieron entonces su capacidad para ayudar, organizar y gestionar una crisis sin ayuda institucional. Es así que el terremoto generó en Tlatelolco una respuesta cívica ante la incapacidad y la corrupción del gobierno, pero además anunció el fin de los ciudadanos como espectadores pasivos. El entonces presidente Miguel de la Madrid manifestó su preocupación de que la participación civil espontánea pudiera convertirse en una amenaza para las instituciones gubernamentales y en su confusión declaró en su momento, que "la sociedad civil era parte del Estado" (Monsiváis, 2008, 1124). Una vez más, el Estado mexicano no pudo aceptar o entender esa participación madura de la ciudadanía tanto en las labores del gobierno como en los asuntos de interés público.

\section{Conclusiones}

Al renunciar a su cargo de embajador en la India como consecuencia de la matanza de Tlatelolco, el escritor Octavio Paz sostenía en una carta que dirigió al diario francés Le Monde: "No es casual que, en los grandes museos de antropología de México, siempre las salas centrales estén dedicadas a los aztecas, es decir a los opresores de la América precolombina, quienes aterrorizaron a los mayas y a

ACE, 14 (42) CC BY-ND 3.0 ES | UPC Barcelona, España | La metacentralidad y sus contradicciones. Tlatelolco como espacio 
los zapotecas. Tampoco es casual que los jóvenes mexicanos asesinados el 2 de octubre hayan caído en la antigua plaza de Tlatelolco: ahí precisamente se encontraba el templo (Teocalli) azteca donde se hacían sacrificios humanos. En ese lugar hoy solamente hay edificios oficiales destinados a los empleados, es decir a la burocracia. El asesinato de los estudiantes fue un sacrificio ritual, porque no existía razón política alguna que justificara ese acto. Sólo se trataba de aterrorizar a la población, usando los mismos métodos de sacrificios humanos de los aztecas" (Redacción, 1995, 15-19).

El lugar donde ocurrieron los hechos ha sido el escenario de una serie de importantes acontecimientos históricos a lo largo de los siglos, desde un lugar paradigmático de los aztecas donde los rituales eran cruentos, pasando por la imposición igualmente violenta de la religión de los conquistadores para lo cual la centralidad y monumentalidad urbana potencializaría su poder simbólico como escenario de la protesta, la rebeldía y la represión. El edificio de la Secretaría de Relaciones Exteriores se encontraba flanqueando la Plaza de las Tres Culturas como una torre de cien metros de altura que representaba la cara de México ante el mundo y, no obstante, desde sus ventanas se apostaron militares y camarógrafos el 2 de día de la masacre. Es interesante el hecho que en 2007 el edificio fuera entregado a la Universidad Nacional Autónoma de México para crear el Centro Cultural Universitario Tlatelolco de la UNAM que preserva la memoria de los eventos de 1968. Los 1.500 anaqueles que custodiaban los archivos oficiales secretos ahora albergarán los archivos conmemorativos de 1968. Este momento por sí solo marca la importancia que la contestación en esos años tuvo en la creación de una ciudadanía emergente, donde una revisión de las relaciones de poder replanteó las políticas de la vida cotidiana urbana. Ante la percepción de las protestas como la creación de un ágora moderno y una nueva apropiación del espacio urbano, hemos argumentado que estos espacios de contestación surgieron a partir de la desesperación y el rechazo del régimen anterior y que tuvieron una función instrumental para cimentar la democracia en el tiempo.

\section{Agradecimientos}

Se agradecen las sugerencias al documento de parte de los pares ciegos, así como el apoyo del programa PRODEP y de la Universidad Autónoma del Estado de Morelos para la publicación de este trabajo.

Conflicto de intereses: El autor declara que no hay conflicto de intereses.

\section{Bibliografía}

Aguayo, S. (2015). De Tlatelolco a Ayotzinapa. Las violencias del Estado. Ciudad de México, México: Proceso.

Bazán, G. (2012). Los rollos perdidos. Ciudad de México, México, documental 90 Min.

De Garay, G. (2000). Mario Pani. Historia oral de la Ciudad de México. Ciudad de México, México: Instituto Mora.

Document 10 (27 de agosto, 1968). Student Disorders. U.S. Embassy in México, confidential telegram. National Archives, RG 59, 1967-69, Pol 13-2 Mex, Box 2340.

Document 14 (23 de septiembre, 1968). George Denney's Conversation with Víctor Urquídi. U.S. Embassy in México, confidential memoranda of conversation. Released to National Security Archive under the Freedom of Information Act, February.

ACE, 14 (42) CC BY-ND 3.0 ES | UPC Barcelona, España | La metacentralidad y sus contradicciones. Tlatelolco como espacio 
Document 25 (5 de noviembre, 1968). Contingency - Scenarios. U.S. Embassy in México, confidential telegram. Fuente: National Archives, RG 59, 1967-69. Pol 13-2 Mex, Box 2340.

Document 32 (29 de Agosto, 1968). Mexican President's Decision to Use Force Against Students May Exacerbate Differences. Bureau of Intelligence and Research, confidential intelligence note. Source: National Archives, RG 59, 1967-69. Pol 23-8 Mex, Box 2343.

Document 35 (26 de septiembre, 1968). Mexican Government's Use of Force Probably Forecloses the Possibility of a Compromise Solution to the Student Conflict. Bureau of Intelligence and Research, confidential intelligence note. Source: National Archives, RG 59, 1967-69. Pol 13-2 Mex, Box 2340.

Document 43 (circa 5 de Noviembre, 1968). Student Violence and Attitudes in Latin America. Bureau of Intelligence and Research, confidential working draft. Source: Lyndon B. Johnson Library, National Security Files Intelligence File, Box 3, "Student Unrest".

Document 44 (28 de marzo, 1968). Security Conditions in México City. CIA, secret intelligence estimate. Source: Lyndon B. Johnson Library, National Security Files National Intelligence Estimates, Box 8, "80/90 Latin America".

Document 77 (4 de octubre, 1968). A Renewed Violence in México. CIA, secret intelligence summary. Source: Released to National Security Archive under the Freedom of Information Act, March 2002.

Document 79 (11 de octubre, 1968). México City Sitrep. CIA Station in México, confidential intelligence information cable. Source: Released to National Security Archive under the Freedom of Information Act, March 2002.

Document 80 (1ero de Noviembre, 1968). Mexican Government Readies for More Student Trouble. CIA, secret intelligence summary. Source: Released to National Security Archive under the Freedom of Information Act, March 2002.

Editorial (1959). México This Month, Vol. V (2), Febrero, 1959, 3.

Essex, S. \& Chalkley, B. (1998). Olympic Games: Catalyst of Urban Change, Leisure Studies 17 (3), 187206.

Foucault, M. (1966). Utopías y Heterotopías. Conferencias radiofónicas entre el 7 y el 21 de diciembre, France-Culture dedicadas a la Literatura y la Utopía.

Guttmann, A. (1992). The Olympics: a history of the modern games. Urbana. IL., EUA: University of Illinois Press.

Guttmann, A. (1994). Games and empires. Modern sports and cultural imperialism. New York, EUA: Columbia University Press.

Harvey, D. (1989). From Managerialism to Entrepreneurialism: The Transformation in Urban Governance in Late Capitalism, Geografiska Annaler. Series B, Human Geography, The Roots of Geographical Change: 1973 to the Present, Vol. 71 (1), 3-17.

Lefebvre, H. (1991). The Production of Space. Oxford \& Cambridge, UK: Blackwell.

López Arretche, L. (1968). El Grito. México 1968. Ciudad de México, Centro Universitario de Cinematografía, 120 Min.

Mendoza, C. (2002). Tlatelolco: Las claves de la masacre. Ciudad de México, México: La Jornada y Canal 6 de Julio, cortometraje 58 Min.

Monsiváis C. (1986). El día del derrumbe y las semanas de la comunidad (De noticieros y de crónicas), Cuadernos Políticos (45), 11-24.

ACE, 14 (42) CC BY-ND 3.0 ES | UPC Barcelona, España | La metacentralidad y sus contradicciones. Tlatelolco como espacio 17 paradoxal de resistencia en la Ciudad de México. DOI: http://dx.doi.org/10.5821/ace.14.42.8224 
Monsiváis C. (1ero de septiembre, 2008). 68: La herencia en busca de herederos. En Revista Proceso. Edición Especial No.23. Tlatelolco 68. La Impunidad, Año 31.

Montemayor, C. (2000). Rehacer la Historia. Análisis de Nuevos Documentos del 2 de octubre de 1968 en Tlatelolco. Ciudad de México, México: Planeta.

Montemayor, C. (2010). La violencia de Estado en México. Antes y después de 1968. Colección Debate, Ciudad de México, México: Random House Mondadori.

National Security Archive, Electronic Briefing Book 99, Document 20, 68. Recuperado de https://nsarchive2.gwu.edu//NSAEBB/NSAEBB204/index.htm\#10

National Security Archive, Electronic Briefing Book 99, Document 1. Recuperado de https://nsarchive2.gwu.edu//NSAEBB/NSAEBB204/index.htm\#10

National Security Archive, Electronic Briefing LITEMPO: The CIA's Eyes on Tlatelolco. CIA Spy Operations in Mexico. Book No. $204 . \quad$ Recuperado de https://nsarchive2.gwu.edu//NSAEBB/NSAEBB204/index.htm\#10

National Security Archive, Electronic Briefing RG 59, 1967-69, Pol 23-8 Mex, Box 2343. Recuperado de https://nsarchive2.gwu.edu//NSAEBB/NSAEBB204/index.htm\#10

National Security Archive, Electronic Briefing, RG 59, 1967-69, Pol 13-2 Mex, Box 2340. Recuperado de https://nsarchive2.gwu.edu//NSAEBB/NSAEBB204/index.htm\#10

Organizing Committee of the Games of the XIX Olympiad (1969). Mexico 68, v.3. The Official Report of the Organizing Committee of the Games of the XIX Olympiad Mexico 1968, Volume 3: The Organization. Recuperado de https://digital.la84.org/digital/collection/p17103coll8/id/23888/

Ortiz Pinchetti, F. (1ero de octubre, 1988). Hernández Toledo, figura de Tlatelolco: una historia de represión. Revista Proceso, Recuperado de https://www.proceso.com.mx/151460/hernandez-toledofigura-de-tlatelolco-una-historia-de-represion

Pani, M. (1952). Los Multifamiliares de Pensiones. Ciudad de México, México: Editorial Arquitectura.

Pani, M. (1960). Conjunto Urbano Nonoalco-Tlatelolco. Regeneración Urbanística de la Ciudad de México, Arquitectura/México 72, 185.

Pani, M. (1966). Tlatelolco, Arquitectura/México, 94/95, 72.

Redacción (24 de septiembre, 1995). Octavio Paz a 'Le Monde' en 1968, Revista Proceso. Recuperado de https://www.proceso.com.mx/170232/octavio-paz-a-le-monde-en-1968

Scherer, J. y Monsiváis, C. (1999). Parte de Guerra, Tlatelolco 1968. Ciudad de México, México: Aguilar.

Sheridan, G. (2015). Habitación con retratos: Ensayos sobre la vida de Octavio Paz. Ciudad de México, México: Era.

Vila, V. (1966). Conjunto Urbano Nonoalco-Tlatelolco. Aspectos Urbanísticos. Arquitectura/México 94/95, 73-105.

ACE, 14 (42) CC BY-ND 3.0 ES | UPC Barcelona, España | La metacentralidad y sus contradicciones. Tlatelolco como espacio 